\title{
GENERALIZED DERIVATION MODULO THE IDEAL OF ALL COMPACT OPERATORS
}

\author{
SALAH MECHERI and AHMED BACHIR
}

Received 3 December 1999

\begin{abstract}
We give some results concerning the orthogonality of the range and the kernel of a generalized derivation modulo the ideal of all compact operators.
\end{abstract}

2000 Mathematics Subject Classification: 47B47, 47A30, 47B20, 47B10.

1. Introduction. Let $\mathscr{L}(\mathscr{H})$ be the algebra of all bounded operators acting on a complex Hilbert space $\mathscr{H}$. For $A$ and $B$ in $\mathscr{L}(\mathscr{H})$, let $\delta_{A, B}$ denote the operator on $\mathscr{L}(\mathscr{H})$ defined by $\delta_{A, B}(X)=A X-X B$. If $A=B$, then $\delta_{A}$ is called the inner derivation induced by $A$. In [1, Theorem 1.7], Anderson showed that if $A$ is normal and commutes with $T$ then, for all $X \in \mathscr{L}(\mathscr{H})$,

$$
\|T-(A X-X A)\| \geq\|T\| .
$$

In [4], we generalized this inequality, we showed that if the pair $(A, B)$ has the Putnam-Fuglede's property (in particular if $A$ and $B$ are normal operators) and $A T=$ $T B$, then for all $X \in \mathscr{L}(\mathscr{H})$,

$$
\|T-(A X-X B)\| \geq\|T\| .
$$

The related inequality (1.1) was obtained by Maher [3, Theorem 3.2] who showed that, if $A$ is normal and $A T=T A$, where $T \in C_{p}$, then $\|T-(A X-X A)\|_{p} \geq\|T\|_{p}$ for all $X \in \mathscr{L}(\mathscr{H})$, where $C_{p}$ is the von Neumann-Schatten class, $1 \leq p<\infty$, and $\|\cdot\|_{p}$ its norm. Here we show that Maher's result is also true in the case where $C_{p}$ is replaced by $\mathscr{K}(\mathscr{H})$, the ideal of all compact operators with $\|\cdot\|_{\infty}$ its norm. Which allows to generalize these results, we prove that if the pair $(A, B)$ has $(\mathrm{PF})_{\mathscr{K}(\mathscr{H})}$, the Putnam-Fuglede's property in $\mathscr{K}(\mathscr{H})$, and $A T=T B$, where $T \in \mathscr{K}(\mathscr{H})$, then $\|T-(A X-X B)\|_{\infty} \geq\|T\|_{\infty}$ for all $X \in \mathscr{L}(\mathscr{H})$.

2. Normal derivations. In this section, we investigate on the orthogonality of the range and the kernel of a normal derivation modulo the ideal of all compact operators. We recall that the pair $(A, B)$ has the property $(\mathrm{PF})_{\mathscr{K}(\mathscr{H})}$ if $A T=T B$, where $T \in \mathscr{K}(\mathscr{H})$ implies $A^{*} T=T B^{*}$. Before proving this result we need the following lemmas.

LEMmA 2.1. Let $N, X \in \mathscr{L}(\mathscr{H})$, where $N$ is a diagonal operator. If $\delta_{N}(X)+S \in \mathscr{K}(\mathscr{H})$, then $S \in \mathscr{K}(\mathscr{H})$ and $\left\|\delta_{N}(X)+S\right\|_{\infty} \geq\|S\|_{\infty}$. 
Proof. Let $\lambda_{1}, \lambda_{2}, \ldots, \lambda_{n}$ be eigenvalues of the diagonal operator $N$. Then, the operator $N$ can be written under the following matrix form:

$$
\left[\begin{array}{cccc}
\lambda_{1} & 0 & \cdots & 0 \\
0 & \lambda_{2} & & \vdots \\
\vdots & & \ddots & 0 \\
0 & \cdots & 0 & \lambda_{n}
\end{array}\right]
$$

According to the following decomposition of $\mathscr{H}$ :

$$
\mathscr{H}=\bigoplus_{i=1}^{n} \operatorname{ker}\left(N-\lambda_{j}\right)
$$

Let $\left|\delta_{i j}\right|$ and $\left|X_{i j}\right|$ be the matrix representations of $S$ and $X$ according to the above decomposition of $\mathscr{H}$. Then

$$
N X-X N=\left|\left(\lambda_{i}-\lambda_{j}\right) X_{i j}\right|
$$

Since $S \in\{N\}^{\prime}$ (the commutant of $N$ ), we get $S_{i j}=0$ for $i \neq j$. Consequently

$$
N X-X N+S=\left[\begin{array}{cccc}
S_{11} & * & * & * \\
* & S_{22} & * & * \\
* & * & * & * \\
* & * & * & S_{n n}
\end{array}\right]
$$

Here $*$ stands for some entry.

As $\delta_{N}(X)+S \in \mathscr{K}(\mathscr{H})$, so $S \in \mathscr{K}(\mathscr{H})$ and the result of Gohberg and Kreĭn [2] guarantee that $\left\|\delta_{N}(X)+S\right\|_{\infty} \geq\|S\|_{\infty}$.

LEMMA 2.2. Let $N \in \mathscr{L}(\mathscr{H})$ be a normal operator and let $\mathscr{H}_{1}=\operatorname{Vect}_{\lambda \in \mathbb{C}} \operatorname{ker}(N-\lambda)$. If $S \in\{N\}^{\prime}$ and there exists $X \in \mathscr{L}(\mathscr{H})$ such that $\delta_{N}(X)+S \in \mathscr{K}(\mathscr{H})$, then $\mathscr{H}_{1}$ reduces $S$ and the restriction $\left.S\right|_{\mathscr{H}_{1}^{\perp}}=0$.

Proof. Since $N$ is a normal operator, $\mathscr{H}_{1}$ reduces $N$ and the restriction $\left.N\right|_{\mathscr{H}_{1}}$ is a diagonal operator, then the Putnam-Fuglede's theorem guarantees that $S^{*} \in\{N\}^{\prime}$. Hence, $\mathscr{H}_{1}$ reduces $S$. Let

$$
N=\left[\begin{array}{cc}
N_{1} & 0 \\
0 & N_{2}
\end{array}\right], \quad S=\left[\begin{array}{cc}
S_{1} & 0 \\
0 & S_{2}
\end{array}\right], \quad X=\left[\begin{array}{ll}
X_{11} & X_{12} \\
X_{21} & X_{22}
\end{array}\right]
$$

on $\mathscr{H}=\mathscr{H}_{1} \oplus \mathscr{H}_{2}$, where $\mathscr{H}_{2}=\mathscr{H}_{1}^{\perp}$. The hypothesis $\delta_{N}(X)+S \in \mathscr{K}(\mathscr{H})$ would imply that $\delta_{N_{2}}\left(X_{22}\right)+S_{2} \in \mathscr{K}(\mathscr{H})$. The result of Anderson [1] (applied to the Calkin algebra $\left.\mathscr{L}\left(\mathscr{H}_{2}\right) \backslash \mathscr{K}\left(\mathscr{H}_{2}\right)\right)$ guarantees that $S_{2} \in \mathscr{K}(\mathscr{H})$. Since the normal operator $N_{2}$ is without eigenvalues and the selfadjoint operator $S_{2}^{*} S_{2}$ is compact and belongs to the commutant of $N_{2}$, it results that $S_{2}^{*} S_{2}=0$ and thus $S_{2}=0$. 
THEOREM 2.3. Let $N \in \mathscr{L}(\mathscr{H})$ be a normal operator, $S \in\{N\}^{\prime}$, and $X \in \mathscr{L}(\mathscr{H})$. If $\delta_{N}(X)+S \in \mathscr{K}(\mathscr{H})$, then $S \in \mathscr{K}(\mathcal{H})$ and

$$
\left\|\delta_{N}(X)+S\right\|_{\infty} \geq\|S\|_{\infty}
$$

Proof. Since $\delta_{N}(X)+S \in \mathscr{K}(\mathscr{H})$, it follows from Lemma 2.2 that

$$
N=\left[\begin{array}{cc}
N_{1} & 0 \\
0 & N_{2}
\end{array}\right], \quad S=\left[\begin{array}{cc}
S_{1} & 0 \\
0 & S_{2}
\end{array}\right]
$$

on $\mathscr{H}=\mathscr{H}_{1} \oplus \mathscr{H}_{1}^{\perp}$, where $\mathscr{H}_{1}=\operatorname{Vect}_{\lambda \in \mathbb{C}} \operatorname{ker}(N-\lambda)$. If

$$
X=\left[\begin{array}{ll}
X_{11} & X_{12} \\
X_{21} & X_{22}
\end{array}\right]
$$

on $\mathscr{H}=\mathscr{H}_{1} \oplus \mathscr{H}_{1}^{\perp}$, then

$$
\delta_{N}(X)+S=\left[\begin{array}{cc}
\delta_{N_{1}}\left(X_{11}\right)+S_{1} & * \\
* & *
\end{array}\right] .
$$

Since $\delta_{N}(X)+S \in \mathscr{K}(\mathscr{H})$, it results that $\delta_{N_{1}}\left(X_{11}\right)+S_{1} \in \mathscr{K}(\mathscr{H})$. As $N$ is a diagonal operator and $S_{1} \in\left\{N_{1}\right\}^{\prime}$, it follows from Lemma 2.1 that $S_{1}$ is compact and

$$
\left\|\delta_{N_{1}}\left(X_{11}\right)+S_{1}\right\|_{\infty} \geq\left\|S_{1}\right\|_{\infty} .
$$

Consequently, $S$ is compact and

$$
\left\|\delta_{N}(X)+S\right\|_{\infty} \geq\left\|\delta_{N_{1}}\left(X_{11}\right)+S_{1}\right\|_{\infty} \geq\left\|S_{1}\right\|_{\infty}=\|S\|_{\infty} .
$$

COROLlary 2.4. Let $N, M, S \in \mathscr{L}(\mathscr{H})$ such that $N$ and $M$ are normal operators and $N S=S M$. If $X \in \mathscr{L}(\mathscr{H})$ such that $\delta_{N, M}(X)+S \in \mathscr{K}(\mathscr{H})$, then $S \in \mathscr{K}(\mathscr{H})$ and

$$
\left\|\delta_{N, M}(X)+S\right\|_{\infty} \geq\|S\|_{\infty}
$$

Proof. Consider the operators $L, T$, and $Y$ defined on $\mathscr{H}=\mathscr{H} \oplus \mathscr{H}$ by

$$
L=\left[\begin{array}{cc}
N & 0 \\
0 & M
\end{array}\right], \quad S=\left[\begin{array}{ll}
0 & S \\
0 & 0
\end{array}\right], \quad Y=\left[\begin{array}{cc}
0 & X \\
0 & 0
\end{array}\right],
$$

then $L$ is normal, $T \in\{L\}^{\prime}$ and

$$
\delta_{L}(Y)+T=\left[\begin{array}{cc}
0 & \delta_{N, M}(X)+S \\
0 & 0
\end{array}\right]
$$

Then Theorem 2.3 would imply that $T$ is compact and

$$
\left\|\delta_{L}(Y)+T\right\|_{\infty} \geq\|T\|_{\infty},
$$


consequently, $S$ is compact and

$$
\left\|\delta_{N, M}(X)+S\right\|_{\infty} \geq\|S\|_{\infty} .
$$

3. Generalized derivations. In this section, we generalize the above results to a large class of operators. We show that if the pair $(A, B)$ has the property $(\mathrm{PF})_{\mathscr{K}(\mathscr{H})}$, and $A S=S B$ such that $\delta_{N, M}(X)+S \in \mathscr{K}(\mathscr{H})$, then $S \in \mathscr{K}(\mathscr{H})$ and

$$
\left\|\delta_{A, B}(X)+S\right\|_{\infty} \geq\|S\|_{\infty}, \quad \forall x \in \mathscr{L}(\mathscr{H}) .
$$

Before proving this result, we need the following lemma.

LEMMA 3.1. Let $A, B \in \mathscr{L}(\mathscr{H})$. The following statements are equivalent:

(1) the pair $(A, B)$ has the property $(\mathrm{PF})_{\mathscr{K}(\mathscr{H})}$;

(2) if $A T=T B$, where $T \in \mathscr{H}(\mathscr{H})$, then $\overline{R(T)}$ reduces $A$, $\operatorname{ker}(T)^{\perp}$ reduces $B$, and $\left.A\right|_{\overline{R(T)}}$ and $\left.B\right|_{\operatorname{ker}(T) \perp}$ are normal operators.

Proof. $(1) \Rightarrow(2)$. Since $\mathscr{K}(\mathscr{H})$ is a bilateral ideal and $T \in \mathscr{K}(\mathscr{H})$, then $A T \in \mathscr{K}(\mathscr{H})$. Hence, as $A T=T B$ and $(A, B)$ satisfies $(\mathrm{PF})_{\mathscr{K}(\mathscr{H})}, A^{*} T=T B^{*}$ and $\overline{R(T)}$, and $\operatorname{ker}(T)^{\perp}$ are reducing subspaces for $A$ and $B$, respectively. Since $A(A T)=(A T) B$ implies $A^{*}(A T)=(A T) B^{*}$ by $(\mathrm{PF})_{\mathscr{K}(\mathscr{H})}$, and the identity $A^{*} T=T B^{*}$ implies that $A^{*} A T=$ $A A^{*} T$, thus we see that $\left.A\right|_{\overline{R(T)}}$ is normal. Clearly, $\left(B^{*}, A^{*}\right)$ satisfies $(\mathrm{PF})_{\mathscr{K}(\mathscr{H})}$ and $B^{*} T^{*}=$ $T^{*} A^{*}$. Therefore, it follows from the above argument that $\left.B^{*}\right|_{\overline{R\left(T^{*}\right)}}=\left.B\right|_{\operatorname{ker}(T)^{\perp}}$ is normal.

(2) $\Rightarrow(1)$. Let $T \in \mathscr{K}(\mathscr{H})$ such that $A T=T B$. Taking the two decompositions of $\mathscr{H}$, $\mathscr{H}_{1}=\mathscr{H}=\overline{R(T)} \oplus \overline{R(T)}{ }^{\perp}$ and $\mathscr{H}_{2}=\mathscr{H}=\operatorname{ker}(T)^{\perp} \oplus \operatorname{ker} T$. Then we can write $A$ and $B$ on $\mathscr{H}_{1}$ into $\mathscr{H}_{2}$, respectively,

$$
A=\left[\begin{array}{cc}
A_{1} & 0 \\
0 & A_{2}
\end{array}\right], \quad B=\left[\begin{array}{cc}
B_{1} & 0 \\
0 & B_{2}
\end{array}\right],
$$

where $A_{1}$ and $B_{1}$ are normal operators. Also we can write $T$ and $X$ on $\mathscr{H}_{2}$ into $\mathscr{H}_{1}$

$$
T=\left[\begin{array}{cc}
T_{1} & 0 \\
0 & 0
\end{array}\right], \quad X=\left[\begin{array}{ll}
X_{1} & X_{2} \\
X_{3} & X_{4}
\end{array}\right]
$$

It follows from $A T=T B$ that $A_{1} T_{1}=T_{1} B_{1}$. Since $A_{1}$ and $B_{1}$ are normal operators, then, by applying the Fuglede-Putnam's theorem, we obtain $A_{1}^{*} T_{1}=T_{1} B_{1}^{*}$, that is, $A^{*} T=T B^{*}$.

THEOREM 3.2. Let $A, B \in \mathscr{L}(\mathscr{H})$ satisfying (PF) $)_{\mathscr{H}}(\mathscr{H})$ and $A S=S B$. If $X \in \mathscr{L}(\mathscr{H})$ such that $\delta_{A, B}(X)+S \in \mathscr{K}(\mathscr{H})$, then $S \in \mathscr{K}(\mathscr{H})$ and

$$
\left\|\delta_{A, B}(X)+S\right\|_{\infty} \geq\|S\|_{\infty}
$$


Proof. Since the pair $(A, B)$ satisfies the property $(\mathrm{PF})_{\mathscr{K}(\mathscr{H})}$, it follows by Lemma 3.1 that $\overline{R(S)}$ reduces $A$, $\operatorname{ker}(S)^{\perp}$ reduces $B$, and $\left.A\right|_{\overline{R(S)}}$ and $\left.B\right|_{\operatorname{ker}(S) \perp}$ are normal operators. Let $\mathscr{H}_{1}=\overline{R(S)} \oplus \overline{R(S)^{\perp}}$ and $\mathscr{H}_{2}=\operatorname{ker}(S)^{\perp} \oplus \operatorname{ker} S$. Then

$$
\begin{array}{ll}
A=\left[\begin{array}{cc}
A_{1} & 0 \\
0 & A_{2}
\end{array}\right], \quad B=\left[\begin{array}{cc}
B_{1} & 0 \\
0 & B_{2}
\end{array}\right], \\
S=\left[\begin{array}{cc}
S_{1} & 0 \\
0 & 0
\end{array}\right], & X=\left[\begin{array}{ll}
X_{1} & X_{2} \\
X_{3} & X_{4}
\end{array}\right] .
\end{array}
$$

It follows from

$$
A S-S B=\left[\begin{array}{cc}
A_{1} S_{1}-S_{1} B_{1} & 0 \\
0 & 0
\end{array}\right]=0
$$

that $A_{1} S_{1}=S_{1} B_{1}$ and we have

$$
\|S-(A X-X B)\|_{\infty}=\left\|\left[\begin{array}{cc}
S_{1}-\left(A_{1} X_{1}-X_{1} B_{1}\right) & * \\
* & *
\end{array}\right]\right\|_{\infty} .
$$

Since $A_{1}$ and $B_{1}$ are two normal operators, then it results from Corollary 2.4 that $S_{1}$ is compact and

$$
\left\|S_{1}-\left(A_{1} X_{1}-X_{1} B_{1}\right)\right\|_{\infty} \geq\left\|S_{1}\right\|_{\infty}
$$

so

$$
\|S-(A X-X B)\|_{\infty} \geq\left\|S_{1}-\left(A_{1} X_{1}-X_{1} B_{1}\right)\right\|_{\infty} \geq\left\|S_{1}\right\|_{\infty}=\|S\|_{\infty}
$$

COROLlary 3.3. Let $A, B \in \mathscr{L}(\mathscr{H})$ satisfying $(\mathrm{PF})_{\mathscr{H}(\mathscr{H})}$ and $A S=S B$. If $X \in \mathscr{L}(\mathscr{H})$ such that $\delta_{A, B}(X)+S \in \mathscr{K}(\mathscr{H})$, then $S \in \mathscr{K}(\mathscr{H})$ and

$$
\|S+A X-X B\|_{\infty} \geq\|S\|_{\infty}
$$

in each of the following cases:

(1) if $A, B \in \mathscr{L}(\mathscr{H})$ such that $\|A x\| \geq\|x\| \geq\|B x\|$ for all $x \in \mathscr{H}$;

(2) if $A$ is invertible and $B$ such that $\left\|A^{-1}\right\|\|B\| \leq 1$.

Proof. (1) The result of Tong [5, Lemma 1] guarantees that the above condition implies that for all $T \in \operatorname{ker}\left(\delta_{A, B} \mid \mathscr{K}(\mathscr{H})\right), \overline{R(T)}$ reduces $A, \operatorname{ker}(T)^{\perp}$ reduces $B$, and $\left.A\right|_{\overline{R(T)}}$ and $\left.B\right|_{\operatorname{ker}(T)^{\perp}}$ are unitary operators. Hence, it results from Lemma 3.1 that the pair $(A, B)$ has the property $(\mathrm{PF})_{\mathscr{K}(\mathscr{H})}$ and the result holds by Theorem 3.2.

Inequality (3.10) holds in particular if $A=B$ is isometric; in other words, $\|A x\|=\|x\|$ for all $x \in \mathscr{H}$.

(2) In this case, it suffices to take $A_{1}=\|B\|^{-1} A$ and $B_{1}=\|B\|^{-1} B$, then $\left\|A_{1} x\right\| \geq$ $\|x\| \geq\left\|B_{1} x\right\|$ and the result holds by (1) for all $x \in \mathscr{H}$. 


\section{REFERENCES}

[1] J. H. Anderson, On normal derivations, Proc. Amer. Math. Soc. 38 (1973), 135-140.

[2] I. C. Gohberg and M. G. Kreĭn, Introduction to the Theory of Linear Nonselfadjoint Operators, Translations of Mathematical Monographs, vol. 18, American Mathematical Society, Rhode Island, 1969.

[3] P. J. Maher, Commutator approximants, Proc. Amer. Math. Soc. 115 (1992), no. 4, 9951000 .

[4] S. Mecheri, On minimizing $\|S-(A X-X B)\|_{p}^{p}$, Serdica Math. J. 26 (2000), no. 2, 119-126.

[5] Y. S. Tong, Kernels of generalized derivations, Acta Sci. Math. (Szeged) 54 (1990), no. 1-2, 159-169.

Salah Mecheri: Department of Mathematics, College of Science, King Saud UniverSITY, P.O. BOX 2455, RIYADH 11451, SAUDI ARABIA

E-mail address: mecherisa1ah@hotmai 1.com

AHMED BACHIR: University OF MOSTAGANEM, EXACTE SCIENCES INSTITUTE, BP 227, 27000 MOSTAGANEM, ALGERIA

E-mail address: bachir_ahmed@hotmai1.com 


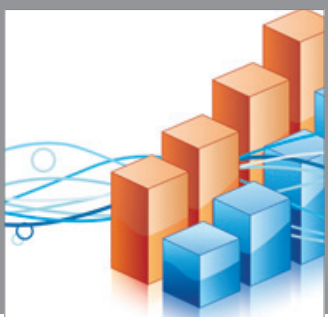

Advances in

Operations Research

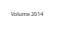

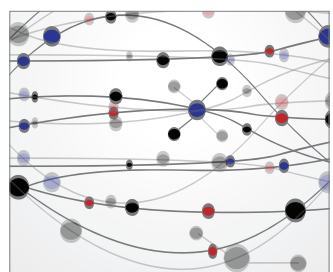

\section{The Scientific} World Journal
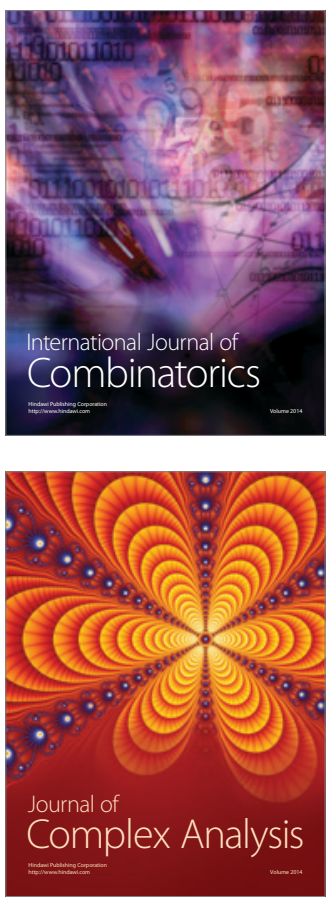

International Journal of

Mathematics and

Mathematical

Sciences
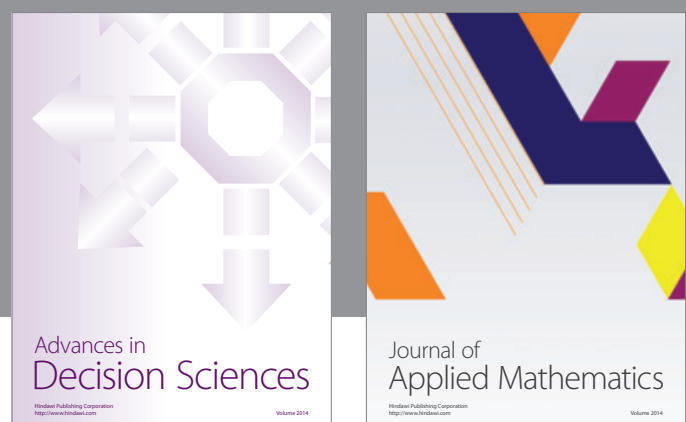

Journal of

Applied Mathematics
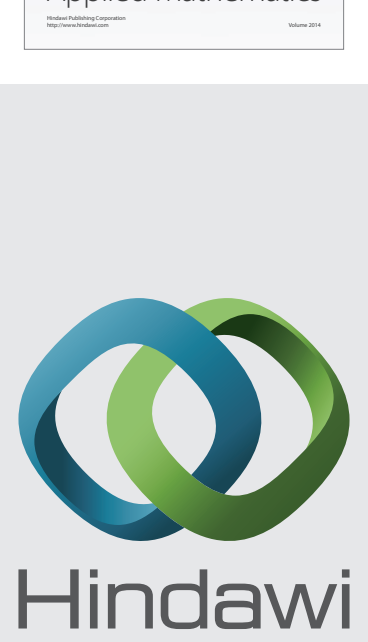

Submit your manuscripts at http://www.hindawi.com
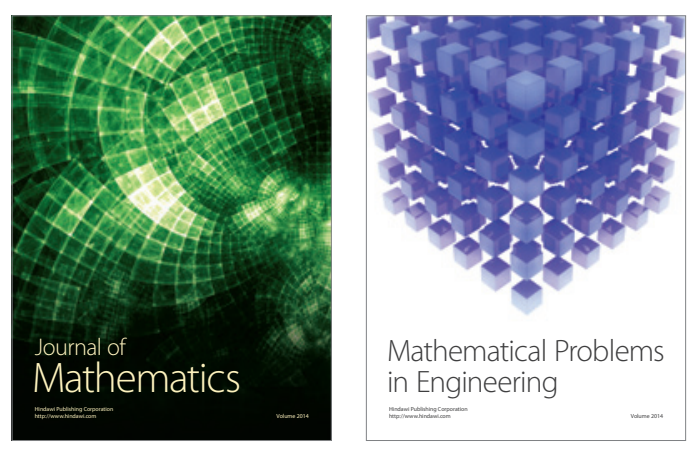

Mathematical Problems in Engineering
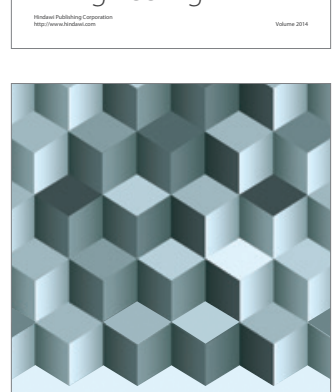

Journal of

Function Spaces
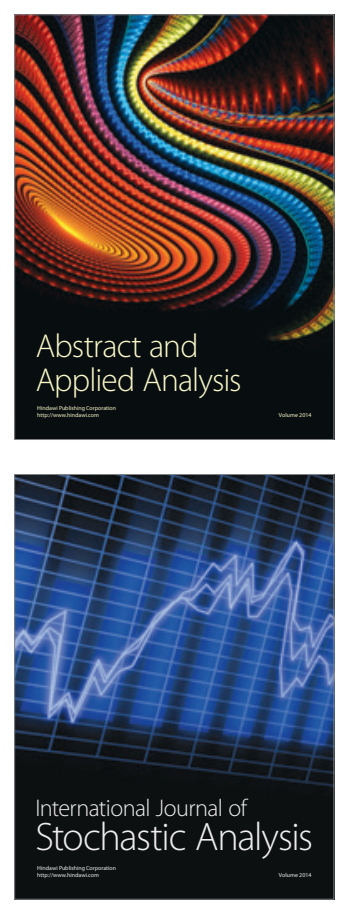

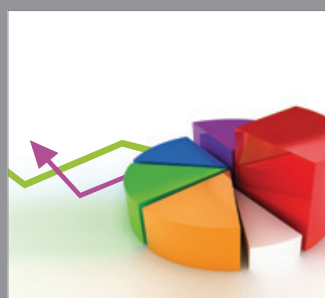

ournal of

Probability and Statistics

Promensencen
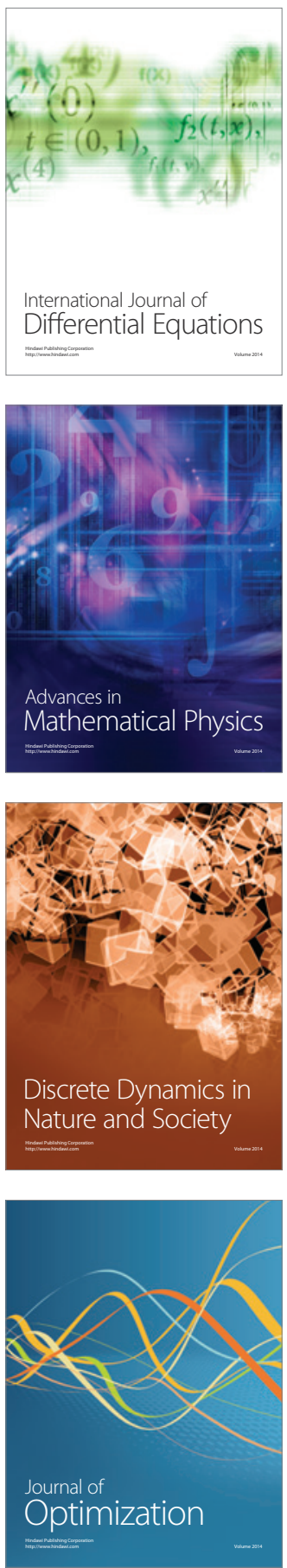